\title{
STELLAR EVOLUTION AND MASS TRANSFER IN BINARIES
}

\author{
A. A. BOJARCHUK \\ Institute of Astronomy, Moscow, Russia. \\ V.M.CHECHETKIN, O.A.KUZNETZOV, YU.P.POPOV \\ Keldysh Institute of Applied Mathematics, \\ Moscow, Russia.
}

ABSTRACT The problem of angular momentum loss in binaries and its influence on stellar evolution is considered. The results of 2D hydrodynamics computer simulation of mass transfer is presented.

More then $30 \%$ stars exist in binary systems. Therefore the evolution of star in interacting binary systems is of great importance for theory of stellar evolution. For example, the Supernova of type IA is appear to occur as a result of evolution in interacting binary system (Iben and Tutukov 1984; Chechetkin et al 1980). The main problem for theory of stellar evolution in binaries is mass transfer and loss of angular momentum. When the stellar envelope reaches its Roche lobe, the star begins to loss the matter and if loss of mass is significant, stellar evolution changes as compared with evolution of single star (Masevich and Tutukov 1988). The size of Roche lobe depends on components mass ratio and distance between the components. Due to loss of angular momentum the distance between components is decreased. This effect can lead to acceleration of stellar evolution.

We present a full hydrodynamical model for simulating of mass transfer in binaries consisting of the giant or subgiant (hereafter the secondary) and a dwarf (hereafter the primary). For instance, we can apply our models for investigation of a star 
wind in Symbiotic stars.

We have chosen the system with parameters:

$$
\begin{aligned}
& \mathbb{N}_{1}=\mathbb{N}_{\odot} \mathbb{R}_{2}=4 \cdot \mathbb{N}_{\odot} \\
& \text { A (distance between components) }=575 \cdot R_{\odot} \\
& \text { orbital velocity of secondary }=8.1 \mathrm{~km} / \mathrm{sec} \\
& R_{2}=191 \cdot R_{\odot} \text { (moderate filling of Roche lobe) }
\end{aligned}
$$

We took the follows boundary conditions on the surface of secondary: $a_{b}=1 \mathrm{~km} / \mathrm{sec}, c_{b}=43 \mathrm{~km} / \mathrm{sec}$. We obtain the stationary shock wave and non-stationary hot tongues behind the primary, moving down-stream. Flow lines shows that it takes particles three revolution to reach the primary and the flow lines that form the disk initiate on a side opposite to the primary (see Fig.1).

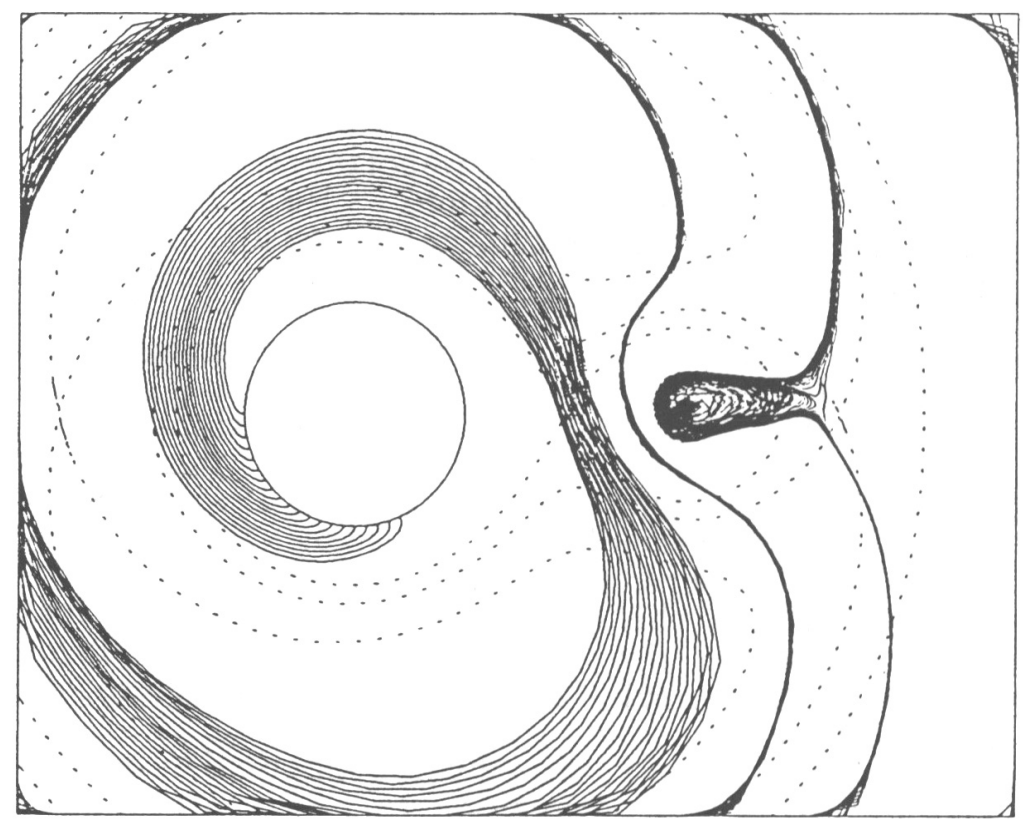

Fig.1

We define the angular momentum as $\mathrm{J}=\Omega \cdot \mathrm{A}^{2} \cdot \mathbb{M}_{1} \cdot \mathbb{M}_{2} / \mathbb{\pi}$ and momentum loss rate as $\mathrm{J}=\mathbb{\pi}_{\mathrm{r}} \cdot \mathrm{r} \cdot \mathrm{V}_{\varphi}$, where $\mathrm{r}$ distance from the center of mass of the system, $V_{\varphi}-$ 
tangential velocity in laboratory system. And we defined the time scale for momentum loss as $\tau=\mathrm{J} / \mathrm{J}$. Fig. 2 represents dependency $\tau(t)$ for input flow from secondary and output flow (far from center mass of the system) for the value of mass loss $\dot{m}=10^{-5} \mathrm{M}_{\odot} /$ year.

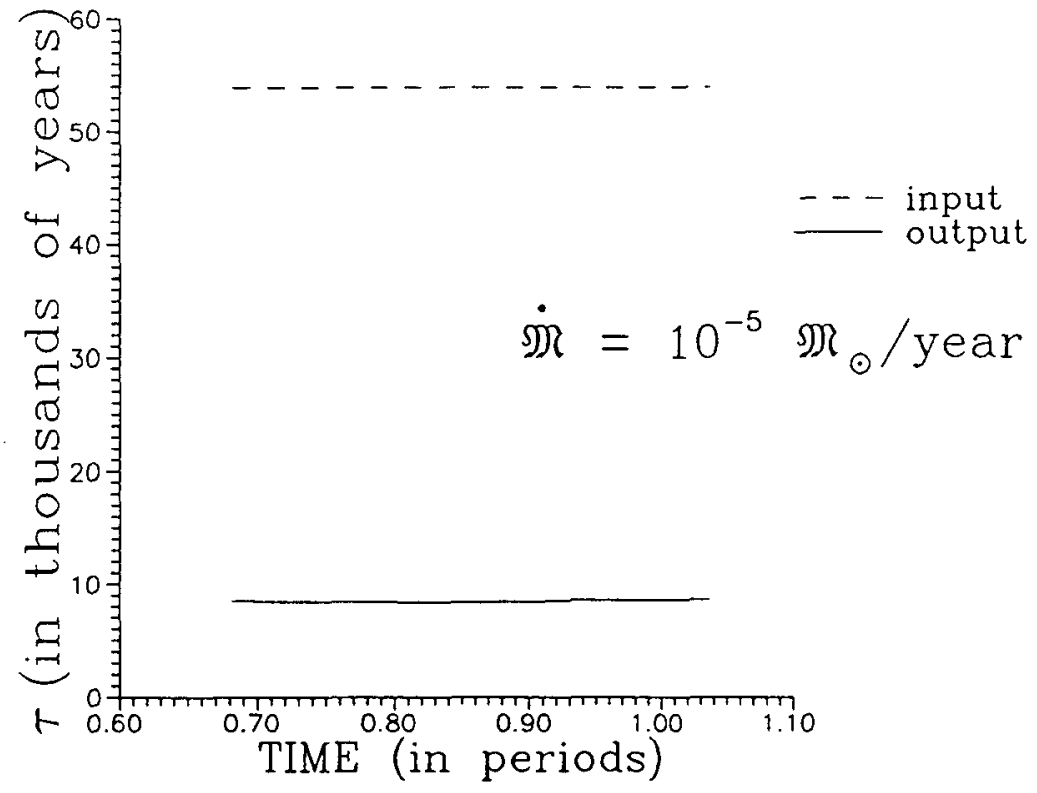

Fig.2

\section{REFERENCES}

Chechetkin,V.M., Gerstein,S.S., Imshennik,V.S., Ivanova, L. N., Khlopov, M. Yu. 1980, Astrophys. and Space Sci., 67, p.61.

Iben, I.,Jr., and Tutukov, A.V. 1984, Ap.J.Suppl., 54, p. 335.

Masevich, A.G. and Tutukov,A.V., 1988, Evolution of Stars - Theory and observation, Moscow, Nauka. 